\title{
Induction and Survival of Binucleated Purkinje Neurons by Selective Damage and Aging
}

\author{
Lorenzo Magrassi, ${ }^{1 *}$ Piercesare Grimaldi, ${ }^{3 *}$ Adalberto Ibatici, ${ }^{4}$ Mirko Corselli, ${ }^{4}$ Laura Ciardelli, ${ }^{2}$ Sandra Castello, ${ }^{4}$ \\ Marina Podestà, ${ }^{4}$ Francesco Frassoni, ${ }^{4}$ and Ferdinando Rossi ${ }^{3}$ \\ ${ }^{1}$ Neurochirurgia Dipartimento Scienze Chirurgiche, Università di Pavia, Fondazione Instituto di Ricovero e Curaio a Carattere Scientifico Policlinico S. \\ Matteo and Instituto di Geneticamolecolare Consiglio Nazionale delle Ricerche, and ${ }^{2}$ Laboratorio Immunologia Neonatale, Laboratorio Sperimentale di \\ Ricerca Area trapiantologica, Fondazione Instituto di Ricovero e Cura a Carattere Scientifico Policlinico S. Matteo, 27100 Pavia, Italy, ${ }^{3}$ Rita Levi Montalcini \\ Center for Brain Repair, National Institute of Neuroscience, Dipartmento di Neuroscienze, Sez. di Fisiologia, Università di Torino, I-10125 Torino, Italy, and \\ ${ }^{4}$ Centro Cellule Staminali e Terapia Cellulare, Divisione di Ematologia, Ospedale San Martino, 16100 Genova, Italy
}

Fusion of bone marrow-derived cells with adult Purkinje cells in the cerebellum gives rise to binucleated Purkinje cells. Whether fusion can be modulated by epigenetic factors and whether fused neurons are stable has remained unclear. Here, we show that in mice and rats, partial ablation of Purkinje cells and local microglial activation in the absence of structural damage to the cerebellum increase the rate of fusion. Moreover, mouse Purkinje cells once fused with bone marrow-derived cells are viable for at least 7 months. We also show that cerebellar irradiation is unnecessary for the generation of binucleated Purkinje cells after bone marrow grafting. Moreover, binucleated Purkinje cells can be found in aged mice that did not receive any treatment, suggesting that fusion events occasionally occur throughout the whole lifespan of healthy, unmanipulated individuals. However, in aged chimeric mice that, after bone marrow transplant, have the majority of their nucleated blood cells fluorescent, the number of binucleated fluorescent Purkinje cells is two orders of magnitude less than the total number of binucleated Purkinje cells. This suggests that, in the majority of heterokaryons, either the incoming nucleus is quickly inactivated or fusion is not the only way to generate a binucleated Purkinje cell.

Key words: Purkinje neurons; bone marrow transplantation; cell fusion; brain repair; heterokaryons; cerebellum

\section{Introduction}

Rare Purkinje neurons expressing enhanced green fluorescent protein (GFP) are detected in the cerebella of mice more than 1 year after total body irradiation followed by infusion of bone marrow-derived cells stably expressing GFP under a strong constitutive promoter (Priller et al., 2001). The accepted explanation for that finding is fusion of donor bone marrow-derived cells with host Purkinje cells, whereas the idea that bone marrowderived cells could transdifferentiate into Purkinje cells is considered unlikely. Evidence supporting fusion derived from experiments where conditional Cre-dependent $\beta$-galactosidase (LacZ) reporter transgenic mice were irradiated and grafted with bone marrow-derived cells constitutively expressing Cre recombinase and GFP (Alvarez-Dolado et al., 2003). The recipient cerebellum contained only LacZ-positive cells and no GFP-positive (green)

\footnotetext{
Received Feb. 3, 2007; revised July 12, 2007; accepted July 16, 2007.

This work was supported by the Ministero dell'Università e della Ricerca Scientifica e Tecnologica (Fondo Integrativo Ricerca di Base RBNE01YRA3-004, PRIN 2005051740-004, 2005), Istituto Superiore di Sanità (Convenzione 530/F-A5), Ministero della Salute (Nuove terapie cellulari per le malattie neurodegenerative), European Community (contract 512039), and Compagnia di San Paolo (Neurotransplant Project 2004.2019). F.F. and F.R. should be considered last authors. We thank M. Okabe (Osaka University, Osaka, Japan) for the generous gift of the enhanced GFP-expressing transgenic mice and transgenic rats.

*L.M. and P.G. contributed equally to this work.

Correspondence should be addressed to Dr. Lorenzo Magrassi, Neurosurgery, Department of Surgical Sciences, University of Pavia, Piazzale Golgi 2, 27100 Pavia, Italy. E-mail: magrassi@igm.cnr.it.

D0I:10.1523/JNEUROSCI.2539-07.2007

Copyright $\odot 2007$ Society for Neuroscience $\quad 0270-6474 / 07 / 279885-08 \$ 15.00 / 0$
}

Purkinje cells. The presence of LacZ-positive Purkinje cells suggests fusion with bone marrow-derived cells resulting in the activation by Cre-mediated recombination of the floxed LacZ reporter in the nucleus of the recipient neurons (Alvarez-Dolado et al., 2003). Further support to fusion came from the observation that, in grafted mice, the majority of Purkinje cells expressing markers of donor origin were binucleated (Alvarez-Dolado et al., 2003; Weimann et al., 2003).

Despite initial observations claiming that green Purkinje cells are only visible 1 year after bone marrow transplant (Priller et al., 2001), later studies have shown that green Purkinje cells can be found after 2 months (Alvarez-Dolado et al., 2003), and their frequency increases linearly with age (Weimann et al., 2003). However, the number of green Purkinje cells compared with the whole population remains extremely low even in long term survivors: 1 year after bone marrow transplantation, there are approximately five green neurons every 10,000 Purkinje cells (Priller et al., 2001).

Altogether, the available data indicate that fusion of bone marrow-derived cells to Purkinje cells after bone marrow transplant does happen, but very little is known about its regulation or its physiological/pathological significance. We do not know, for example, whether the frequency of fusion can be modified, whether irradiation of the cerebellum before bone marrow grafting is instrumental to allow fusion, or whether fused neurons persist or quickly degenerate. 
Here, we demonstrate the following: (1) limited selective damage to host Purkinje cells increases the number of binucleated green neurons; (2) irradiation of the cerebellum is not necessary to induce fusion between bone marrow-derived cells and Purkinje cells; (3) Purkinje cell heterokaryons can survive for months; (4) comparing the number of binucleated Purkinje neurons in the cerebella of unmanipulated mice of different ages, we suggest that fusion is an ongoing phenomenon occurring spontaneously and physiologically in aged mice; and (5) in the cerebella of mice that survived 15-18 months after bone marrow transplantation with $>85 \%$ of GFP-positive nucleated cells in their peripheral blood, binucleated Purkinje cells outnumber almost two orders of magnitude the number of binucleated green Purkinje cells. This last observation can indicate either a high rate of nuclear inactivation in Purkinje cell heterokaryons or an alternative mechanism to fusion with bone marrow-derived cells for the generation of binucleated Purkinje cells.

\section{Materials and Methods}

Bone marrow transplant

Animals. In all experiments involving mice, the recipients of bone marrow transplantation were C57BL/6 (H-2b/CD45.2) mice. Bone marrowderived cells for transplantation were obtained from 5- to 10 -week-old male transgenic mice derived by outcrossing the original "green mice" (Okabe et al., 1997) as described previously (Magrassi et al., 2003). Those donor mice ubiquitously express GFP. Nonfluorescent donors used for the second transplant in the double bone marrow transplantation experiments came from 5- to 8-week-old male C57BL/6 (H-2b/CD45.2) mice.

Recipient rats were Sprague Dawley, and bone marrow-derived cells were obtained from GFP-expressing rats of the same strain (Ito et al., 2001).

Each experimental group comprised initially at least 20 mice or rats. The number of mice reaching final analysis varied according to each experimental group, and it will be indicated in the results sections for each experimental group ( $n$, number of animals reaching final analysis).

Conditioning and graft. Mice and rats used as recipients of bone marrow transplants were conditioned by irradiation with a total dose of 900 cGy (mice) or 1000 cGy (rats) from a ${ }^{60}$ Co source 24 h before transplantation. Recipient mice conditioned by alkylating-based chemotherapy regimen received Treosulfan (Medac, Wedel, Germany) at myeloablative doses (7000 mg/kg total dose) and Fludarabine at $600 \mathrm{mg} / \mathrm{kg}$ (Schering, Berlin, Germany). Treosulfan was dissolved in sterile water $\left(27^{\circ} \mathrm{C}\right)$ at a final concentration of $66.6 \mathrm{mg} / \mathrm{ml}$ and injected intraperitoneally twice in $1 \mathrm{~d}$ in volumes of 0.15 or $0.175 \mathrm{ml} / 10 \mathrm{~g}$ body weight (1000 and 1165 $\mathrm{mg} / \mathrm{kg}$, respectively) on days 3, 2, and 1 before transplantation. Fludarabine was dissolved in sterile saline solution at a final concentration of 50 $\mathrm{mg} / \mathrm{ml}$ and administered intraperitoneally in volumes of $0.4 \mathrm{ml} / 10 \mathrm{~g}$ body weight $(200 \mathrm{mg} / \mathrm{kg})$ on days 3,2 , and 1 . Four mice treated as described above were killed $5 \mathrm{~d}$ after drug administration to assess their potential toxicity on Purkinje cells. The other mice underwent bone marrow transplantation.

Donor mice were killed by cervical dislocation, their femur and tibias were removed, and marrow was flushed out of the bones with serum-free glucose-supplemented Earle medium (Invitrogen, San Giuliano Milanese, Italy) under sterile conditions. We obtained the suspension of bone marrow-derived cells for grafting by mechanical dissociation of the marrow fragments. After washing three times and centrifugation, donor cells were resuspended in Earle medium to a final concentration of $10^{6}$ cells in $300 \mu$ l.

In all recipient animals either irradiated or treated by treosulfan/fludarabine, the unfractionated marrow cell suspension was grafted by tailvein injection. Donor engraftment was determined by flow cytometry of peripheral blood. Cytometric analysis was performed on days 20 and 40 after bone marrow grafting and on the day the animals were killed. Mice showing a donor chimerism $<20 \%$ on day 40 were intravenously injected with $5 \times 10^{6}$ donor splenocytes. Donor splenocytes were prepared by mincing the spleen. The filtered cell suspension was adjusted to $20 \times$
$10^{7}$ cells per $\mathrm{ml}$ in $10 \mathrm{ml}$ of PBS, and $5 \mathrm{ml}$ aliquots were overlaid on $5 \mathrm{ml}$ of Lympholyte-M (Cedarlane Laboratories, Hornby, Ontario, Canada) and centrifuged at $1450 \times g$ for $20 \mathrm{~min}$ at room temperature.

Surgical procedures. All surgical procedures and perfusions were performed under deep general anesthesia obtained by intraperitoneal administration of ketamine (100 mg/kg Ketavet; Bayer, Leverkusen, Germany) supplemented by xylazine ( $5 \mathrm{mg} / \mathrm{kg}$ Rompun; Bayer). The experimental plan was designed according to the European Communities Council Directive of 1986 (86/609/EEC), National Institutes of Health guidelines, and the Italian law for care and use of experimental animals (DL116/92).

Purkinje cell lesions. Partial degeneration of Purkinje cells was induced by intraventricular injection of toxic substances that selectively affect these neurons [i.e., saporin-conjugated anti-P75 (192 IgG) antibodies (Heckers et al., 1994) or propidium iodide (PI) (Rossi et al., 1993)]. In rats, $2 \mu \mathrm{l}$ of saporin-conjugated 192 IgG (Millipore, Billerica, MA) dissolved in PBS at the final concentration of $1.1 \mathrm{mg} / \mathrm{ml}$ was injected into the right lateral ventricle through a Hamilton syringe [coordinates from bregma: anteroposterior (AP), $1 \mathrm{~mm}$; mediolateral (ML), $\pm 1.5 \mathrm{~mm}$; dorsoventral (DV), $3.5 \mathrm{~mm}$ ). In mice, $1 \mu \mathrm{l}$ of PI (Sigma-Aldrich Italia, Milan, Italy) dissolved in PBS at the final concentration of $1 \mathrm{mg} / \mathrm{ml}$ was injected into the right lateral ventricle through a Hamilton syringe (coordinates from bregma: AP, $0.5 \mathrm{~mm} ; \mathrm{ML}, \pm 1 \mathrm{~mm}$; DV, $1.5 \mathrm{~mm}$ ). Purified GFP $(1 \mathrm{mg} / \mathrm{ml})$ was injected into the cisterna magna of adult mice through a 25 gauge needle by an open suboccipital approach.

\section{Histology and tissue analysis}

Harvesting and processing of the tissues. Mice were killed at various times after bone marrow transplant or at various ages. Under deep general anesthesia, recipient animals were transcardially perfused with $500 \mathrm{ml}$ of $4 \%$ paraformaldehyde (Sigma-Aldrich Italia) in $0.12 \mathrm{M}$ phosphate buffer, $\mathrm{pH}$ 7.2-7.4. The brains were removed, stored overnight in the same fixative at $4^{\circ} \mathrm{C}$, and finally transferred in $30 \%$ sucrose in $0.12 \mathrm{M}$ phosphate buffer. The fixed cerebella were processed in one of two ways. For frozen cryostat sections, the tissues were placed in 30\% sucrose in PBS overnight, embedded in Tissue-Tek OCT (VWR International, Milano, Italy), and serially cut on a cryostat at $30 \mu \mathrm{m}$. For paraffin sections, the tissues were dehydrated and embedded in paraffin wax according to standard techniques and serial sectioned to $7 \mu \mathrm{m}$.

Immunohistochemistry. Cryostat sections were immunohistochemically processed with anti-calbindin antibodies to label Purkinje cells (1: 1500, monoclonal or polyclonal; Swant, Bellizona, Switzerland) and antiGFP antibodies (1:700, polyclonal or monoclonal; Clontech, Pero, Italy) to enhance the GFP fluorescent signal. In addition, to rule out the possibility that chemotherapy treatment directly affected Purkinje cells, cerebellar sections from mice killed $5 \mathrm{~d}$ after drug administration were labeled by antibodies against c-Jun (polyclonal, 1:1000; Santa Cruz Biotechnology, Santa Cruz, CA) or activated caspase 3 (rabbit polyclonal 1:250; Promega, Madison, WI), or by nicotinamide adenine dinucleotide phosphate (NADPH) diaphorase histochemistry (Zagrebelsky et al., 1998).

Incubation of cerebellar slices with primary antibodies was made overnight at room temperature in PBS with $1.5 \%$ normal serum and $0.25 \%$ Triton X-100. The sections were then exposed for $1 \mathrm{~h}$ at room temperature to secondary biotinylated antibodies followed by a solution of streptavidin Texas Red conjugate (1:200; Invitrogen) or fluoresceinated secondary antibody (1:200; Vector Laboratories, Burlingame, CA). Nuclei were counterstained with PI (Sigma-Aldrich Italia). To reduce RNA staining in the cytoplasm, we sometimes incubated in RNase the sections before PI staining. The sections were mounted on microscope slides with Tris-glycerol supplemented with 10\% Mowiol (Calbiochem, La Jolla, CA) to reduce fading of fluorescence.

Microscopical analysis. Stained sections were examined under a Zeiss (Oberkochen, Germany) Axiophot light microscope, equipped with a Nikon (Melville, NY) DS-5M digital camera. Selected sections were also examined with an Olympus Optical (Hamburg, Germany) Fluoview 300 confocal microscope. Digital images were processed with Adobe Photoshop 6.0 (Adobe Systems, San Jose, CA) to adjust contrast and assemble the final plates. Quantitative and morphometric evaluations were made 
using the Neurolucida software (MicroBrightField, Colchester, VT) connected to an E-800 Nikon microscope via a color CCD camera.

Cell counting. Green Purkinje cells were counted directly by complete examination of all serial sections comprehensive of the entire cerebellum.

The number of binucleated Purkinje cells in aged mice was estimated by inspection under the confocal microscope of as many high-power fields ( $100 \times$ oil immersion objective) were necessary to count at least 110 Purkinje cells. Each field was randomly chosen from nonadjacent serial vibratome sections of the entire cerebellum. The sections were stained by an anti-calbindin antibody and counterstained with PI to identify the presence of one or more nuclei in the Purkinje cells.

To estimate the number of Purkinje cells in control and Fludarabine plus Treosulfan treated cerebella, the number of Purkinje cells along 1 $\mathrm{mm}$ of Purkinje cell layer was evaluated on calbindin immunostained sections, according to a previously established method (Buffo et al., 1997).

Flow cytometry. Peripheral blood was subjected to red cell lysis by treatment with ammonium chloride, washed once, and then resuspended in PBS. The relative percentage of bone marrow-derived cells of donor origin was directly distinguished by their intrinsic GFP fluorescence using one-color flow cytometry (FACS Calibur; BD Biosciences, San Jose, CA).

Western blot analysis. Western blot analysis was performed according to standard methods. Plasma was obtained by centrifugation of heparinized blood collected from puncture of the eye plexus, and cerebral spinal fluid was collected by direct puncture of the cisterna magna with a 25 gauge needle. Twelve microliters of CSF and $1 \mu \mathrm{l}$ of plasma were loaded for SDS-PAGE. After transfer to nitrocellulose, the blots were blocked in $10 \%$ nonfat dry milk in Tris-buffered saline and Tween 20 and incubated in the primary antibody (1:100, mouse monoclonal; Clontech) for $2 \mathrm{~h}$ at room temperature. After washing with Tris-buffered saline and Tween 20 , membranes were exposed to horseradish peroxidase-conjugated secondary antibody for $1 \mathrm{~h}$ at room temperature. Immunoreactivities were detected using the enhanced chemiluminescence method (Super Signal West Dura; Pierce, Celbio Pero, Italy) according to the instructions of the manufacturer.

Microfluorimetry. GFP concentration in plasma was determined using a fluorescence spectrometer (LS50B; PerkinElmer, Emeryville, CA), with excitation set at $488 \mathrm{~nm}$ and emission measured at $507 \mathrm{~nm}$. To evaluate the amount of GFP in plasma and CSF, the fluorescence of the samples was compared with standard dilution curves generated by adding known amounts of purified GFP, respectively, to the plasma of wild-type mice or to PBS.

\section{Results}

Damage to Purkinje cells increases fusion events

Chimeric mice were generated by grafting marrow from mice ubiquitously expressing GFP (Okabe et al., 1997; Magrassi et al. 2003 ). Mice showing $>85 \%$ of green fluorescent bone marrowderived cells in their peripheral blood received a single injection of PI into the right lateral ventricle $30-45 \mathrm{~d}$ after transplant. This substance diffuses into the CSF, and it is selectively taken up by Purkinje cells (Borges et al., 1985). PI uptake induces degeneration of approximately one-third of the Purkinje cells within 1 week without apparent damage to other neurons in the cerebellar cortex (Rossi et al., 1993).

Green Purkinje cells were detected in the cerebellum of treated animals 2 weeks after PI injection, thus $\sim 2$ months after bone marrow transplant. On the contrary, in mice that only received bone marrow grafting, we never found green Purkinje cells before 3 months. Three months after intraventricular injection of PI, the average number of green Purkinje cells in the cerebellum was 15.75 ( $\pm 10.9 \mathrm{SD} ; n=4)$ and increased to 105.06 ( $\pm 58.7 \mathrm{SD} ; n=$ 18) at 9 months (Fig. 1). At the same survival times, in mice submitted to bone marrow transplantation not followed by PI administration, we only found an average of $0.6( \pm 0.8 \mathrm{SD} ; n=5)$ and 7 ( $\pm 7.3 \mathrm{SD} ; n=11$ ) green Purkinje cells (Fig. 1 ). Comparable

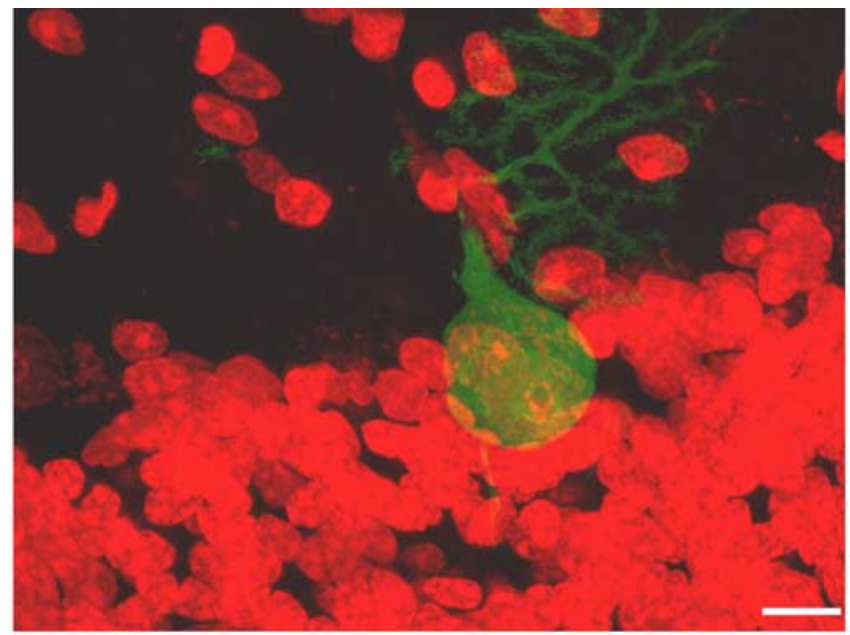

a

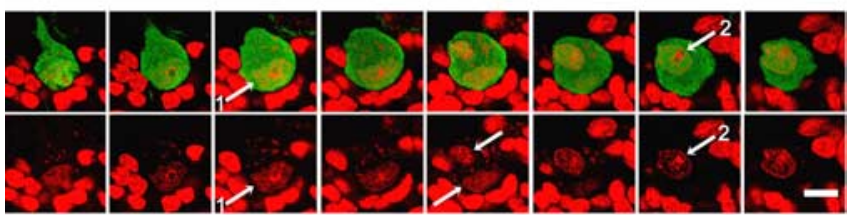

a1

Mice

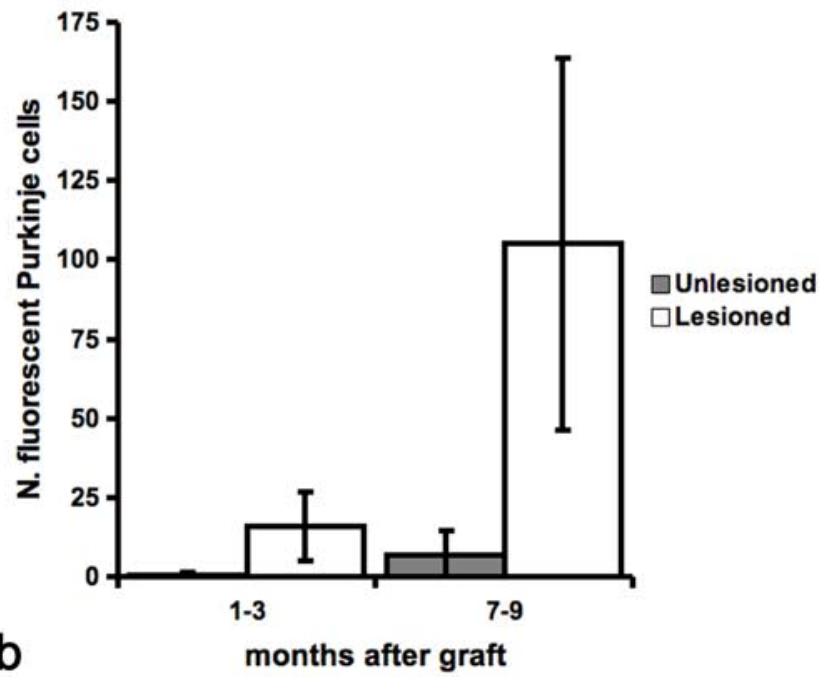

Figure 1. Binucleated green Purkinje cells in the cerebellum of mice after bone marrow grafting and injection of PI. $\boldsymbol{a}$, Confocal image of a binucleated green Purkinje cell in mice cerebellum 9 months after bone marrow transplant. Cell nuclei are stained in red with PI. In a1 the presence of two nuclei (arrows and numbers) is demonstrated by an optical series of the same field. Scale bars, $10 \mu \mathrm{m} . \boldsymbol{b}$, Quantification of green Purkinje cells in PI-treated (lesioned) or untreated (unlesioned) mice 1-3 or 7-9 months after bone marrow transplantation. Notice that, after comparable survival times, the number of green Purkinje cells is one order of magnitude higher in mice treated by Pl injection than in unlesioned mice. The number of green Purkinje cells in lesioned mice also rises faster and earlier than in controls. Error bars represent SD.

results were obtained in rats where incomplete Purkinje cell loss was induced by intraventricular administration of the immunotoxin 192 IgG-saporin (192 IgG) targeting the p75NGF receptor (Heckers et al., 1994). Again, the number of green Purkinje cells increased over time in both groups but was always at least one order of magnitude greater in the immunotoxin-treated animals (Fig. 2). Three months after injection of 192 IgG, the average 


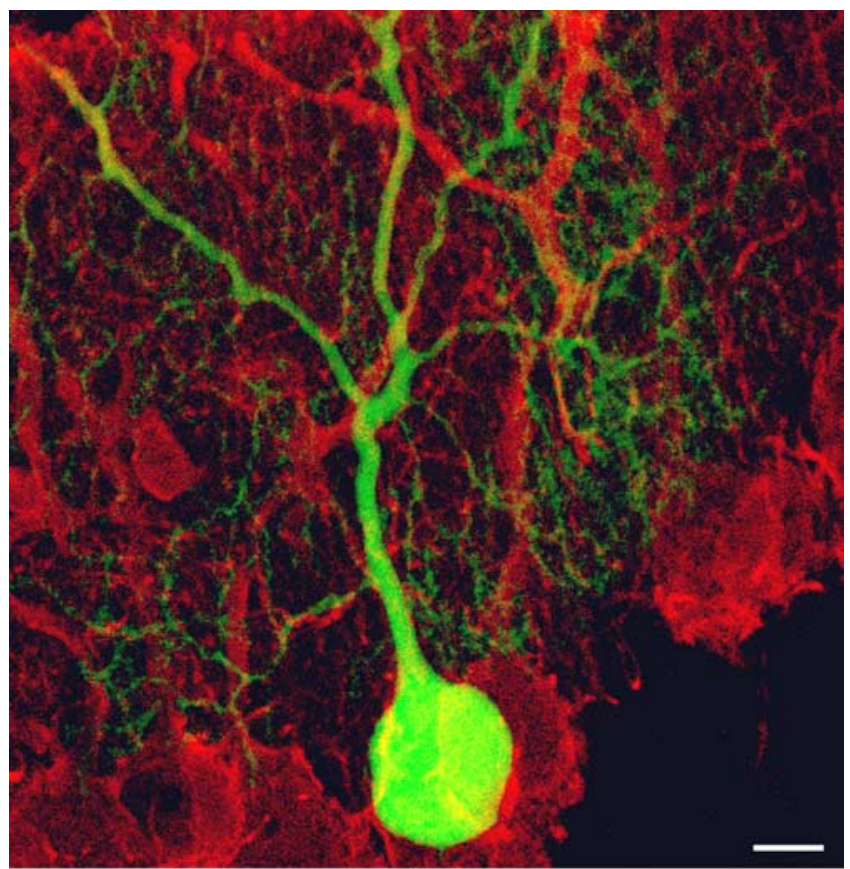

a

\section{Rats}

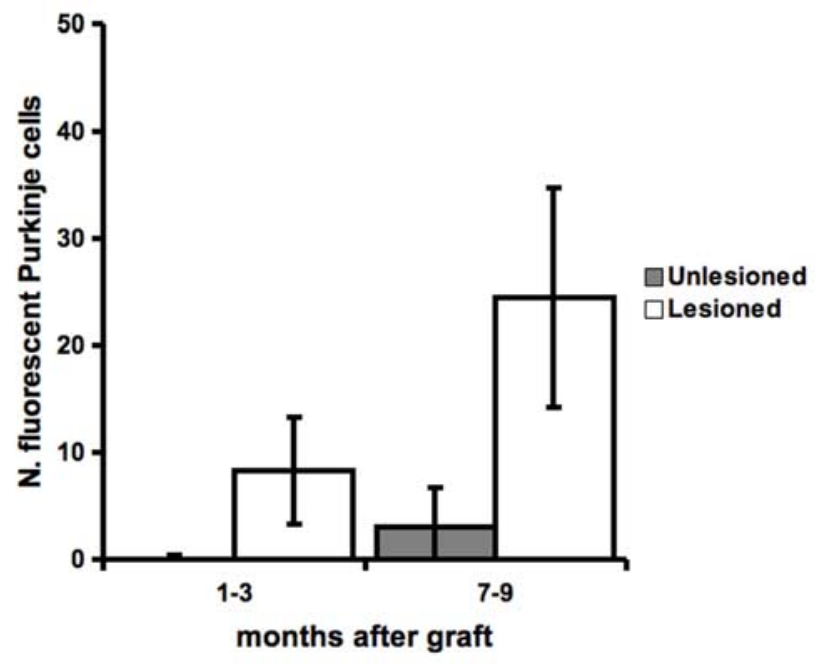

b

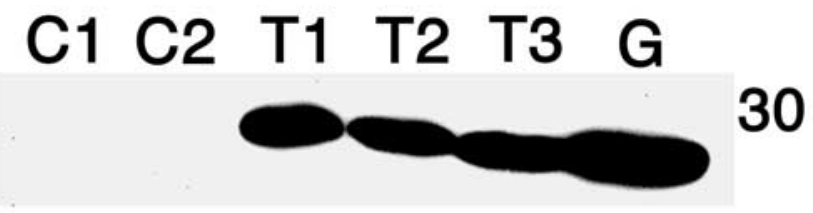

C

Figure 2. Green Purkinje cells in the cerebellum of rats after bone marrow grafting and partial lesioning of the resident Purkinje cells by intraventricular injection of $192 \mathrm{lgG}$. $\boldsymbol{a}$, Confocal image of green Purkinje cells in rat cerebellum 9 months after bone marrow transplantation; Purkinje cells were immunostained for calbindin (red). Scale bar, $10 \mu \mathrm{m}$. $\boldsymbol{b}$, Quantification of green Purkinje cells in lgG 192-treated (lesioned) or untreated (unlesioned) rats 1-3 or 7-9 months after bone marrow transplantation. The number of green Purkinjecells is one order of magnitude higher and increases faster in treated rats than in their unlesioned counterparts. Error bars represent SD. c, Western blot analysis, anti-GFP polyclonal antibody. GFP protein is detected in plasma of three grafted mice $(\mathrm{T} 1, \mathrm{~T} 2$, and $\mathrm{T} 3$ ) and of a donor green mouse (G); no GFP is detected in the corresponding $C S F$ ( $C 1$ and $(2)$. We loaded $1 \mu \mathrm{l}$ of plasma and $12 \mu \mathrm{l}$ of CSF. number of green Purkinje cells was $8.25( \pm 5.0 \mathrm{SD} ; n=3)$, and this value increased to 24.5 ( $\pm 10.3 \mathrm{SD} ; n=7)$ at 9 months. In rats submitted to bone marrow transplantation only, we found an average of $0.14( \pm 0.4 \mathrm{SD} ; n=7)$ and $3.00( \pm 3.7 \mathrm{SD} ; n=4)$ green Purkinje cells after the same survival times.

In mice and rats, accumulation in the cerebellum of microglial cells was spatially limited and restricted in time to the first month after damage induction; thereafter, the cerebellum was not the site of chronic inflammation, and gliosis was also scarce. Because Purkinje cells actively accumulate molecules present in the CSF (Borges et al., 1885), we asked whether green Purkinje cells could result from uptake and accumulation of GFP flowing through the cerebral ventricles. We evaluated by Western blot and spectrofluorimetry the level of free GFP in serum and CSF of mice ( $n=4)$ carrying $>95 \%$ green fluorescent bone marrow-derived cells. We found detectable free GFP in plasma but not in the CSF of those mice (Fig. 2). Furthermore, we injected $2 \mu \mathrm{g}$ of purified GFP dissolved in $5 \mu \mathrm{l}$ of saline into the cisterna magna of three mice submitted to intraventricular administration of PI $2 \mathrm{~d}$ before. One week after GFP injection, the animals were perfused and their cerebella serially cut and inspected for the presence of green Purkinje cells before and after immunostaining with fluoresceinated anti-GFP antibody. We did not find any green Purkinje cells in the cerebella of those animals.

\section{Cerebellar irradiation is dispensable to fusion of Purkinje cells with bone marrow-derived cells}

Total body irradiation is considered the standard conditioning regimen before bone marrow transplantation. In our experiments, mice were irradiated with $900 \mathrm{cGy}$, a level of radiation that generates permanent changes in the CNS and cerebellum (Manda et al., 2006). As shown in the above experiments, damage to Purkinje cells increases the number of green neurons and shortens the delay of their appearance after bone marrow grafting. In vitro exposure of neurons to radiation as low as 200 cGy may induce apoptosis (Gobbel et al., 1998) and in vivo local inflammatory changes with microglial activation (Chiang et al., 1993). Fusion between bone marrow-derived cells and host Purkinje cells may thus only be possible after the initial damage caused by radiation therapy. Furthermore, whole body radiation induces changes in the levels of circulating chemokines that are known to alter the rate of conversion of bone marrow-derived cells to muscle (Abedi et al., 2005). To test whether irradiation of the cerebellum was instrumental in the fusion process, we repeated some of the above experiments by conditioning mice with Treosulfan and Fludarabine instead of total body irradiation. Treosulfan is a derivative of busulfan that did not show appreciable CNS toxicity (Scheulen et al., 2000; Weissert et al., 2003), whereas neurotoxic effects of Fludarabine were observed only at higher doses and spared the cerebellum (Spriggs et al., 1986). To further rule out the possibility that these drugs induced direct damage to the cerebellum and particularly to Purkinje cells, sections from animals killed $5 \mathrm{~d}$ after treatment were labeled for different markers of Purkinje cell stress or death, such as c-Jun, activated caspase 3, and NADPH-diaphorase histochemistry (Zagrebelsky et al., 1998). No differences were observed between treated and control cerebella (data not shown). In addition, in calbindin immunostained sections, Purkinje cells never displayed the typical morphological features associated with degenerative events (Rossi et al., 1995), and there were no signs of cell loss. The number of Purkinje cells per millimeter of Purkinje cell layer length was 45.5 in control mice $-( \pm 1.9 \mathrm{SD} ; n=4)$ and 45.4 in chemo-ablated animals ( $\pm 3.5 \mathrm{SD} ; n=4)$.

Each mouse received Treosulfan and Fludarabine starting $48 \mathrm{~h}$ before bone marrow transplantation. The donor cells were obtained 
from the bone marrow of GFP-expressing mice, and the recipients were C57BL/6. With this protocol, we obtained average values of green nucleated cells in blood after 1 month that were close to $50 \%$. As in the irradiated mice, we injected PI into the lateral cerebral ventricle 30-45 d after bone marrow transplantation to obtain a partial depletion of Purkinje cells in the host cerebellum. Despite the absence of radiation damage to the cerebellum, we found green Purkinje cells in the grafted animals as early as 1 week after administration of PI. Three months after lesioning, the average number of green Purkinje cells in the cerebellum was $2.0( \pm 2.9 \mathrm{SD} ; n=6)$ and increased to $17.5( \pm 14.8 \mathrm{SD} ; n=3)$ at 7 months. The last values were comparable with an average of $4.0( \pm 1.4 \mathrm{SD} ; n=3)$ and $20.5( \pm 7.8$ $\mathrm{SD} ; n=6)$ green Purkinje cells found after the same survival times in irradiated animals with a similar percentage $(50 \%)$ of circulating fluorescent donor cells (Fig. 3).

\section{Once generated green Purkinje cells are stable for at least 7 months}

Chimeric mice with $>85 \%$ circulating fluorescent bone marrowderived cells were generated and submitted to intraventricular injection of PI 30-45 d later. Forty-five to $60 \mathrm{~d}$ after PI injection, the animals were divided into three groups. Those in the first group were immediately killed and the number of green Purkinje cells counted. Those in the second group were kept as controls without additional treatment, whereas mice in the last group were again submitted to total body irradiation followed $24 \mathrm{~h}$ later by another graft of nonfluorescent bone marrow-derived cells obtained from syngenic mice. Animals in the last two groups were fixed by perfusion 7-9 months after the first transplant.

The cerebella of the animals surviving the second total body irradiation and bone marrow transplant, which showed $<5 \%$ circulating fluorescent nucleated cells, were analyzed 7-9 months after the first transplantation. In all cases, we found fluorescent Purkinje cells. The average number of green Purkinje cells in these mice was 25.3 ( $\pm 11.2 \mathrm{SD} ; n=3$ ), which was much lower than 105.06 ( $\pm 58.7 \mathrm{SD} ; n=18$ ) green Purkinje cells counted in mice perfused at the same survival time after a single irradiation and graft of fluorescent bone marrow-derived cells (Fig. 4). Nevertheless, it was comparable with 15.75 ( $\pm 10.6 \mathrm{SD} ; n=4)$ green Purkinje cells found in the cerebella of mice killed at the time when their counterparts underwent the second transplantation of nonfluorescent bone marrow-derived cells. Therefore, in doubly grafted mice, the green Purkinje cells generated between the first and second graft remain viable for at least 7 months.

\section{Binucleated Purkinje cells are present in healthy, unmanipulated 18-month-old mice}

The original description of green Purkinje cells after bone marrow transplantation concerned the cerebella of more than 1-year-old mice in the absence of any lesion to the host Purkinje cells with the exception of the initial whole body irradiation (Priller et al., 2001). Our previous experiments showed that irradiation of the host cerebellum and of the whole body can be completely omitted and still binucleated green Purkinje cells obtained. We speculated that fusion between bone marrow-derived cells and Purkinje neurons could be a physiological phenomenon, which occurs in the complete absence of bone marrow graft, radiation, or any other direct injury to the cerebellum. Therefore, we analyzed by confocal microscopy serial vibratome sections of cerebella obtained from mice kept under standard laboratory conditions for 2 or 18 months. Purkinje cells were stained by an anti-calbindin antibody and counterstained with PI to identify the presence of one or more nuclei (Fig. 5). The results of our analysis indicate an average of $4.97( \pm 2.1 \mathrm{SD} ; n=4)$ doubly nucle-

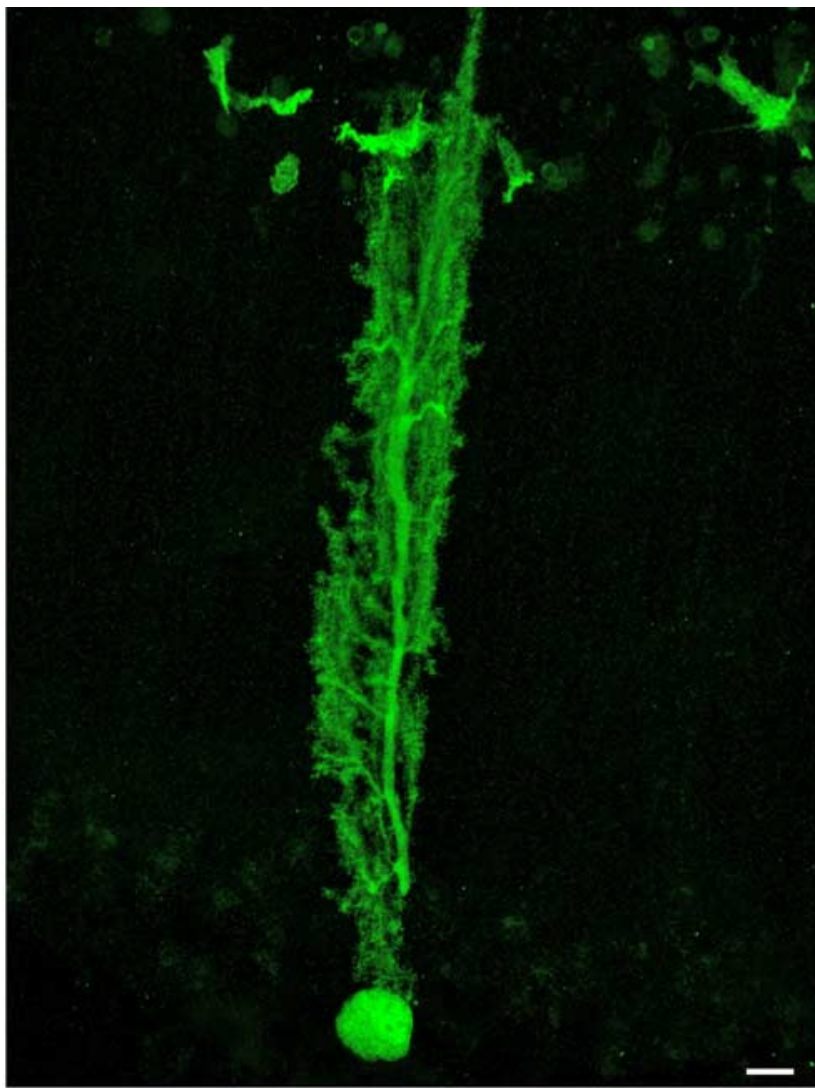

a

\section{Mice}

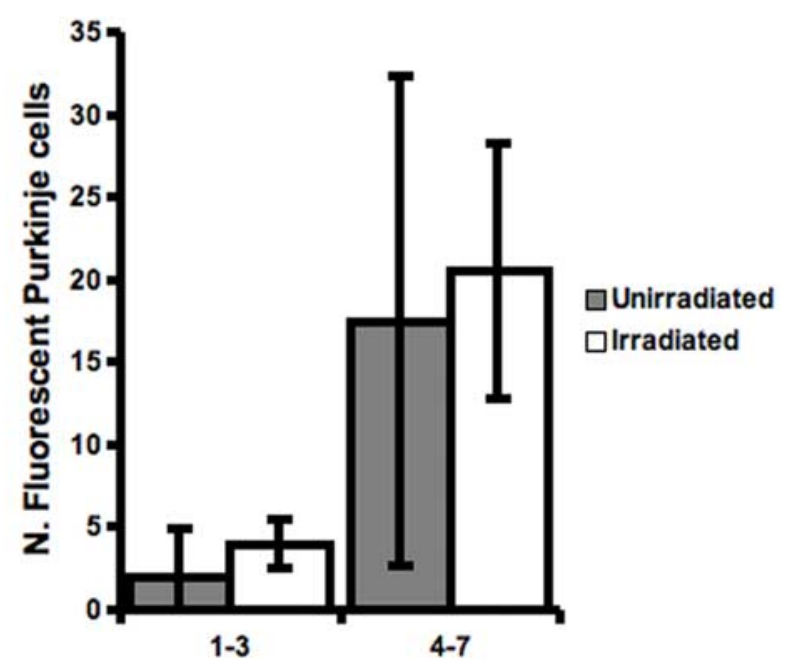

Months from PI injection

b

Figure 3. Green Purkinje cells in the cerebellum of mice in which depletion of the host bone marrow was induced either by intraperitoneal injection of Treosulfan and Fludarabine (Unirradiated) or by whole body irradiation (Irradiated). The average number of fluorescent bone marrow-derived cells in peripheral blood obtained after chemical conditioning was lower $(\sim 50 \%)$ than for those mice described in Figure 1; for comparison, we selected among the irradiated mice those with a comparable percentage of circulating fluorescent cells. All mice of the two groups received an intraventricular injection of $\mathrm{PI} \mathrm{30-45} \mathrm{d} \mathrm{after} \mathrm{grafting.} a$, Confocal image of a green Purkinje cell 7 months after chemical conditioning and bone marrow transplantation. Only cells containing GFP are shown; smaller cells are microglial cells. Scale bar, 10 $\mu \mathrm{m} . \boldsymbol{b}$, Histogram showing that the numbers of green Purkinje cells in the cerebella of the animals in the two groups are comparable. Error bars represent SD. 


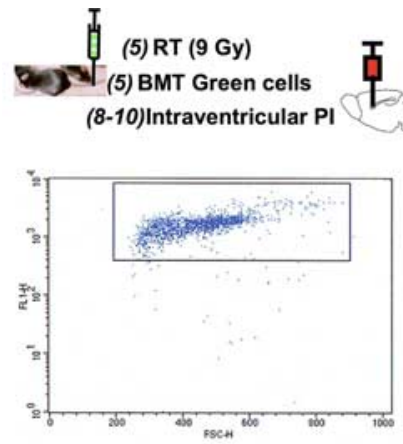

a

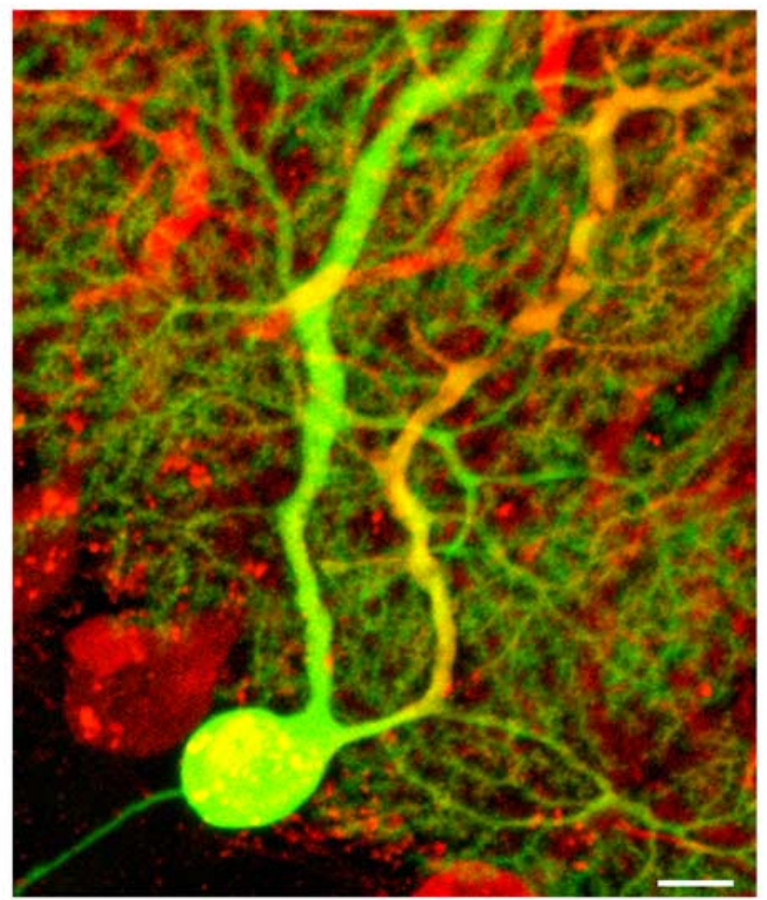

C

\section{Regrafting}

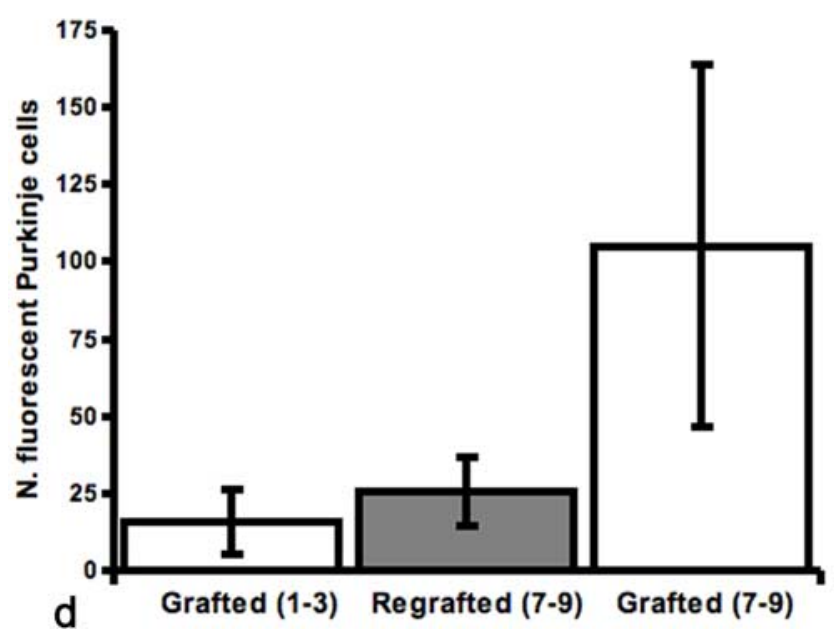

Figure 4. Long-term survival of fluorescent Purkinje cells in the host cerebellum; an outline of the procedures precedes the pictures. The numbers in parenthesis indicate weeks from birth. $\boldsymbol{a}$, Five-week-old C57BL/6 mice were subjected to whole body irradiation and grafted with fluorescent bone marrow-derived cells. Thirty to $45 \mathrm{~d}$ later, after determining the number of circulating green cells by fluorescence-activated cell-sorting analysis, mice were intraventricularly injected with PI. All mice submitted to PI injection had at least $85 \%$ of circulating fluorescent bone marrow-derived cells and many, as shown in $\boldsymbol{a}$, had $>95 \%$. $\boldsymbol{b}$, Forty-five to $60 \mathrm{~d}$ after ated Purkinje cells every 100 Purkinje cells in aged mice. We did not find binucleated Purkinje cells in 2-month-old animals.

Binucleated Purkinje cells greatly outnumber green binucleated Purkinje cells in mice with $>85 \%$ of green nucleated peripheral blood cells

We also analyzed the number of binucleated Purkinje cells in animals submitted to bone marrow transplantation with GFPpositive donor cells. We limited our analysis to long-term surviving (15-18 months of age) mice with $>80 \%$ circulating fluorescent bone marrow-derived cells. On average, we found 3.41 ( \pm $1.8 \mathrm{SD} ; n=3$ ) binucleated Purkinje cells every 100 (corresponding approximately to 9000 binucleated Purkinje cells in the entire cerebellum). The total number of green Purkinje cells we found after serial sectioning and analysis of the entire cerebellum of mice surviving to bone marrow transplantation for comparable times was on average 29.62 ( $\pm 10.7 \mathrm{SD} ; n=7)$. Therefore, in those grafted mice, the frequency of binucleated Purkinje cells was similar to that observed in age-matched unmanipulated mice, but it was more than two orders of magnitude higher than the number of green Purkinje cells.

\section{Discussion}

Fusion of Purkinje cells and bone marrow-derived cells occurs after whole body irradiation and grafting of cells harvested from transgenic mice expressing GFP in all cells of the body (Alvarez-Dolado et al., 2003; Weimann et al., 2003). In that experimental paradigm, green Purkinje cells are binucleated (Weimann et al., 2003).

In all previous studies, irradiation of the cerebellum shortly preceded bone marrow grafting (Priller et al., 2001; Alvarez-Dolado et al., 2003; Weimann et al., 2003). In similar experimental paradigms, whole body radiation increased the rate of conversion of bone marrow-derived cells into muscle (Abedi et al., 2005). Irradiation of the brain temporarily opens the blood-brain barrier, increases the level of inflammation by activation of glial cells (Chiang et al., 1993), and depresses adult neurogenesis (Mizumatsu et al., 2003). Fusion between bone marrow-derived cells and Purkinje neurons may thus be possible only after brain irradiation. Our results show similar numbers of green Purkinje cells in irradiated mice and in mice receiving Treosulfan and Fludarabine as bone marrow conditioning treatment, when comparable levels of circulating fluorescent cells were obtained. Thus, both brain and total body irradiation are unnecessary to induce fusion between bone marrow-derived cells and Purkinje neurons.

Nevertheless, we also show that partial damage to the host Purkinje cells, and that after microglial activation, increase the number of green Purkinje cells after bone marrow grafting even in the absence of mechanical/structural damage to the cerebellum or to the blood-brain barrier. To avoid any direct mechanical injury to the

$\leftarrow$

PI injection (16-18 weeks of age), some of the initial mice were submitted to a second total body irradiation and $24 \mathrm{~h}$ later to a new graft of nonfluorescent syngenic bone marrow cells. The regrafted mice quickly showed a dramatic drop in circulating fluorescent cells: those that showed $<5 \%$, as shown in $\boldsymbol{b}$, were perfused and examined $7-9$ months after the first irradiation and bone marrow graft. c, Confocal image of a green Purkinje cell in the cerebellum of a mouse that underwent double transplantation and was perfused 9 months after the first graft. Purkinje cells were immunostained for calbindin (red). Scale bar, $10 \mu \mathrm{m}$. d, Histogram comparing green Purkinje cells in mice submitted to single (white bars) or double (gray bars) irradiation and transplantation procedures. All mice received a single intraventricular injection of $\mathrm{PI}$ $30-45 \mathrm{~d}$ after the initial graft. The number of green Purkinje cells in regrafted mice 7-9 months after the initial graft is significantly $(p<0.05)$ lower than in singly grafted mice surviving for the same period but similar to that observed in animals killed 1-3 months after a single graft. 

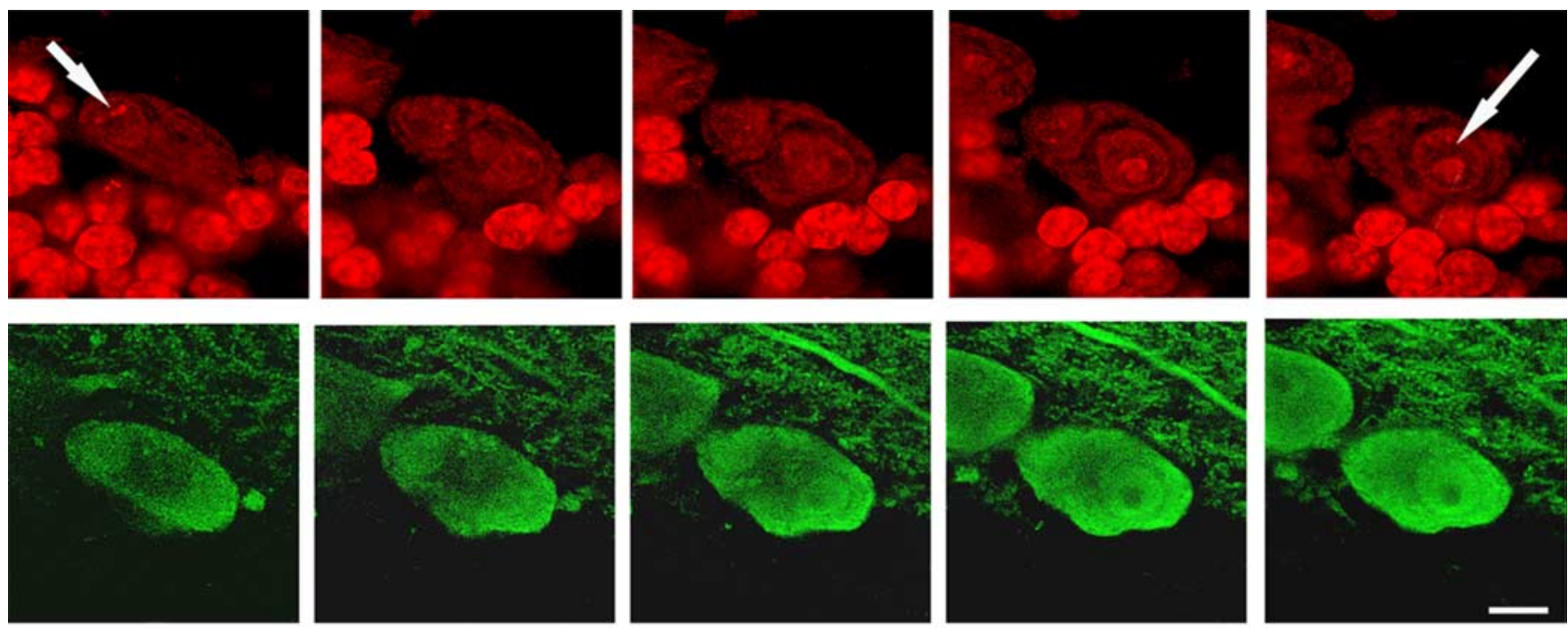

a

Figure 5. Binucleated Purkinje cells are present in the cerebellum of aged mice maintained under standard laboratory conditions. $\boldsymbol{a}$, Confocal images of a binucleated Purkinje cell stained with an anti-calbindin antibody and counterstained by Pl in the cerebellum of an 18-month-old mouse. Scale bar, $10 \mu \mathrm{m}$. Arrows indicate the two nuclei in the same Purkinje cell.

cerebellar tissue, we injected PI or 192 IgG-saporin into the lateral ventricle of mice or rats with various degrees of chimerism for circulating fluorescent cells. Purkinje cells accumulate PI in their cell bodies, and many of them die (Rossi et al., 1993). The immunotoxin 192 IgG-saporin is internalized by those Purkinje cells expressing p75NGF receptor, resulting in selective but incomplete Purkinje cell loss (Heckers et al., 1994). Starting 1 week after these treatments, binucleated green Purkinje cells can be detected in the cerebellum of mice and rats. Since then, the number of green Purkinje cells increases even beyond the end of the period of cell death and local inflammation induced by the toxins.

In BALB/c npcnih (NPC) mice, a model of human NiemannPick disease type $\mathrm{C} 1$, Purkinje cells degenerate starting at the age of 3 weeks (Tanaka et al., 1988). Grafting directly into the cerebellum of these mice, mesenchymal stem cells carrying wild-type acid sphingomyelinase and expressing GFP also resulted in an increased number of fused cells compared with wild-type mice receiving the same transplant (Bae et al., 2005). However, in NPC mice, the damage is not selective to Purkinje cells and nonneuronal cells are also affected. Furthermore, injection of donor cells directly into the cerebellum greatly perturbs the normal structure of the tissue and increases the local concentration of bone marrow-derived cells to levels that are not comparable with those reached after orthotopic transplant. Therefore, in that model, the increased frequency of cell fusion cannot be unequivocally attributed to Purkinje cell damage. Conversely, the results of our experiments, in which there is no direct injury to the cerebellar tissue, bone marrow-derived cells exclusively come from circulation, and Purkinje cells are selectively affected by PI or 192IgG, clearly indicate that damage to Purkinje cells themselves and local microglial accumulation favor the formation of heterokaryons.

Purkinje cells actively accumulate molecules present in the CSF (Borges et al., 1985); we asked whether the increased number of fluorescent Purkinje cells after the injection of PI into the ventricles could be caused by uptake and accumulation of free GFP by Purkinje cells rather than by an increased rate of fusion. However, we detected free GFP only in serum and not in CSF even after PI injection. Furthermore, we did not find any green Purkinje cells after injection of purified GFP into CSF by direct puncture of the cisterna magna.
If cell fusion results from neuronal damage together with local tissue reaction, heterokaryons might be nothing more than a transient condition that shortly precedes degeneration and dismissal of the cell. However, our double transplantation experiments indicate that the majority of fused Purkinje cells are stable and survive at least 7 months after the fusion event. In those experiments, the host Purkinje cells had the opportunity to fuse with fluorescent bone marrow-derived cells only for 3 months after the first transplant (two after damage of Purkinje cells by PI injection) when the number of circulating fluorescent cells greatly exceeded the number of nonfluorescent host cells (chimeric mice had always $>85 \%$ circulating green nucleated cells). After the second irradiation, the number of circulating fluorescent cells dropped to $<5 \%$, making it unlikely any new fusion of green bone marrow-derived cells with host Purkinje cells. As expected, the average number of fluorescent Purkinje cells in the doubly grafted mice 9 months after the initial transplant was comparable with that of animals killed 3 months after a single graft of fluorescent cells (i.e., the period occurring between the first and the second grafts). That observation suggests that fusion of fluorescent bone marrow-derived cells and Purkinje cells continues for the entire life of the animal, as long as the number of circulating fluorescent cells remains high. In addition, the persistence of green Purkinje cells with extremely low or absent circulating fluorescent bone marrow-derived cells indicates that the majority of the cells that underwent fusion in the period between the first and second graft survived and maintained normal morphology for many months.

We counted $\sim 5 \%$ of binucleated Purkinje cells every 100 Purkinje cells in the cerebellum of aged mice (18 months) that survived and were healthy under standard laboratory conditions, whereas we did not find binucleated Purkinje cells in mice aged 2 months. Binucleated Purkinje cells have been described and forgotten in aged rats (Inukai, 1928), senescent mice (Andrew, 1937), and a variety of human pathological conditions (Andrew, 1939). The number of binucleated Purkinje cells reported by Andrew (1939) for aged mice agrees with that found in our study. Age-related Purkinje cell death as shown by a decrease in total Purkinje cell number (Sturrock, 1989a,b; Sturrock, 1990) might favor fusion between those cells and bone marrow-derived cells. However, uncertainties regarding the timing and extent of age 
related Purkinje cell death in the mouse (Zanjani et al., 2004) preclude any direct comparison with our data on the number of binucleated cells in aged mice.

The number of binucleated cells in mice surviving irradiation and bone marrow grafting for 16-18 months was similar to that found in untreated mice, $\sim 3$ in every 100 Purkinje cells. On the contrary, the higher number of binucleated green Purkinje cells we found in those mice was 47 , as directly counted after serial sectioning of the entire cerebellum. Those mice received the bone marrow graft at $\sim 5$ weeks of age and still had $>80 \%$ fluorescent circulating nucleated cells at the time of perfusion. Therefore, if all binucleated cells derived from fusion events, we should expect similar numbers of binucleated green Purkinje cells and binucleated Purkinje cells. In contrast, the two numbers differed by almost two orders of magnitude.

In a previous study, 2-4 months after grafting, mice expressing a floxed version of the lacZ gene with bone marrow cells harvested from donor mice expressing both GFP and Cre recombinase, and LacZ-positive and GFP-negative Purkinje cells were found in the host cerebellum (Alvarez-Dolado et al., 2003). In contrast, in the same mice, the majority of LacZ-positive cells in the liver and heart were also GFP positive. Furthermore, GFPpositive microglial cells were always detected in the brain of the host mice at all survival times (Alvarez-Dolado et al., 2003). Those findings together with our observation that the number of binucleated cells in the cerebellum of aged mice greatly outnumbers the number of GFP-positive Purkinje cells suggest that in the majority of Purkinje cell heterokaryons, the fused nucleus is quickly inactivated. Alternative explanations that cannot be ruled out will be that other cells (e.g., macroglial cells) that are not derived from donor cells can also fuse with Purkinje cells or that fusion is not the only way to generate binucleated Purkinje cells. For instance, DNA duplication and nuclear division without cell division could produce such binucleated neurons.

In any case, our results indicate that occasional cell fusion spontaneously occurs in the lifetime of healthy animals. The fused cells are viable surviving for months to the fusion event and the frequency of these events increases in pathological conditions. The rate of such spontaneous events is low and its physiologic importance unknown. Nevertheless, if we can understand and master the mechanism underlying cell fusion, we might exploit this phenomenon to introduce young nuclei or functional genes in aged or degenerating Purkinje cells and, possibly, in other neuron types.

\section{References}

Abedi M, Greer DA, Foster BM, Colvin GA, Harpel JA, Demers DA, Pimentel J, Dooner MS, Quesenberry PJ (2005) Critical variables in the conversion of marrow cells to skeletal muscle. Blood 106:1488-1494.

Alvarez-Dolado M, Pardal R, Garcia-Verdugo JM, Fike JR, Lee HO, Pfeffer K, Lois C, Morrison SJ, Alvarez-Buylla A (2003) Fusion of bone-marrowderived cells with Purkinje neurons, cardiomyocytes and hepatocytes. Nature 425:968-973.

Andrew W (1937) The effects of fatigue due to muscular exercise on the Purkinje cells of the mouse, with special reference to the factor of age. Ztschr f Zellforschung u mikr Anat 27:534-555.

Andrew W (1939) Origin and significance of binucleate Purkinje cells in man. Arch Pathol 28:821-826.

Bae JS, Furuya S, Shinoda Y, Endo S, Schuchman EH, Hirabayashi Y, Jin HK (2005) Neurodegeneration augments the ability of bone marrow-derived mesenchymal stem cells to fuse with Purkinje neurons in Niemann-Pick type C mice. Hum Gene Ther 16:1006-1011.

Borges LF, Elliot PJ, Gill R, Iversen SD, Iversen LL (1985) Selective extraction of small and large molecules from the cerebrospinal fluid by Purkinje neurons. Science 228:346-348.

Buffo A, Holtmaat AJDG, Savio T, Verbeek S, Oberdick J, Oestreicher AB, Gispen
WH, Verhaagen J, Rossi F, Strata P (1997) Targeted overexpression of the neurite growth-associated protein B-50/GAP-43 in cerebellar Purkinje cells induces sprouting in response to axotomy, but does not allow axon regeneration into growth permissive transplants. J Neurosci 17:8778-8791.

Chiang CS, McBride WH, Withers HR (1993) Radiation-induced astrocytic and microglial responses in mouse brain. Radiother Oncol 29:60-68.

Gobbel GT, Bellinzona M, Vogt AR, Gupta N, Fike JR, Chan PH (1998) Response of postmitotic neurons to X-irradiation: implications for the role of DNA damage in neuronal apoptosis. J Neurosci 18:147-155.

Heckers S, Ohtake T, Wiley RG, Lappi DA, Geula C, Mesulam MM (1994) Complete and selective cholinergic denervation of rat neocortex and hippocampus but not amygdala by an immunotoxin against the p75 NGF receptor. J Neurosci 14:1271-1289.

Inukai T (1928) On the loss of Purkinje cells, with advancing age, from the cerebellar cortex of the albino rat. J Comp Neurol 45:1-31.

Ito T, Suzuki A, Imai E, Okabe M, Hori M (2001) Bone marrow is a reservoir of repopulating mesangial cells during glomerular remodeling. J Am Soc Nephrol 12:2625-2635.

Magrassi L, Castello S, Ciardelli L, Podesta M, Gasparoni A, Conti L, Pezzotta S, Frassoni F, Cattaneo E (2003) Freshly dissociated fetal neural stem/ progenitor cells do not turn into blood. Mol Cell Neurosci 22:179-187.

Manda K, Ueno M, Moritake T, Anzai K (2006) Radiation-induced cognitive dysfunction and cerebellar oxidative stress in mice: protective effect of alpha-lipoic acid. Behav Brain Res 176.

Mizumatsu S, Monje ML, Morhardt DR, Rola R, Palmer TD, Fike JR (2003) Extreme sensitivity of adult neurogenesis to low doses of $\mathrm{X}$-irradiation. Cancer Res 63:4021-4027.

Okabe M, Ikawa M, Kominami K, Nakanishi T, Nishimune Y (1997) "Green mice" as a source of ubiquitous green cells. FEBS Lett 407:313-319.

Priller J, Persons DA, Klett FF, Kempermann G, Kreutzberg GW, Dirnagl U (2001) Neogenesis of cerebellar Purkinje neurons from gene-marked bone marrow cells in vivo. J Cell Biol 155:733-738.

Rossi F, Borsello T, Vaudano E, Strata P (1993) Regressive modifications of climbing fibres following Purkinje cell degeneration in the cerebellar cortex of the adult rat. Neuroscience 53:759-778.

Rossi F, Jankovski A, Sotelo C (1995) Target neuron controls the integrity of afferent axon phenotype: a study on the Purkinje-cell climbing fiber system in cerebellar mutant mice. J Neurosci 15:2040-2056.

Scheulen ME, Hilger RA, Oberhoff C, Casper J, Freund M, Josten KM, Bornhauser M, Ehninger G, Berdel WE, Baumgart J, Harstrick A, Bojko P, Wolf HH, Schindler AE, Seeber S (2000) Clinical phase I dose escalation and pharmacokinetic study of high-dose chemotherapy with treosulfan and autologous peripheral blood stem cell transplantation in patients with advanced malignancies. Clin Cancer Res 6:4209-4216.

Spriggs DR, Stopa E, Mayer RJ, Schoene W, Kufe DW (1986) Fludarabine phosphate (NSC 312878) infusions for the treatment of acute leukemia: phase I and neuropathological study. Cancer Res 46:5953-5958.

Sturrock RR (1989a) Changes in neuron number in the cerebellar cortex of the ageing mouse. J Hirnforsch 30:499-503.

Sturrock RR (1989b) Age related changes in Purkinje cell number in the cerebellar nodulus of the mouse. J Hirnforsch 30:757-760.

Sturrock RR (1990) A comparison of quantitative histological changes in different regions of the ageing mouse cerebellum. J Hirnforsch 31:481-486.

Tanaka J, Nakamura H, Miyawaki S (1988) Cerebellar involvement in murine sphingomyelinosis: a new model of Niemann-Pick disease. J Neuropathol Exp Neurol 47:291-300.

Weimann JM, Johansson CB, Trejo A, Blau HM (2003) Stable reprogrammed heterokaryons form spontaneously in Purkinje neurons after bone marrow transplant. Nat Cell Biol 5:959-966.

Weissert R, Wiendl H, Pfrommer H, Storch MK, Schreiner B, Barth S, Seifert T, Melms A, Dichgans J, Weller M (2003) Action of treosulfan in myelinoligodendrocyte-glycoprotein-induced experimental autoimmune encephalomyelitis and human lymphocytes. J Neuroimmunol 144:28-37.

Zagrebelsky M, Buffo A, Skerra A, Schwab M, Strata P, Rossi F (1998) Retrograde regulation of growth-associated gene expression in adult rat Purkinje cells by myelin-associated neurite growth inhibitory proteins. J Neurosci 18:7912-7929.

Zanjani H, Lemaigre-Dubreuil Y, Tillakaratne NJ, Blokhin A, McMahon RP, Tobin AJ, Vogel MW, Mariani J (2004) Cerebellar Purkinje cell loss in aging Hu-Bcl-2 transgenic mice. J Comp Neurol 475:481-492. 